\title{
Papers
}

\section{Randomised controlled trial of butterbur and cetirizine for treating seasonal allergic rhinitis}

Andreas Schapowal on behalf of Petasites Study Group

\begin{abstract}
Objectives To compare the efficacy and tolerability of butterbur (Petasites hybridus) with cetirizine in patients with seasonal allergic rhinitis (hay fever).

Design Randomised, double blind, parallel group comparison.

Setting Four outpatient general medicine and allergy clinics in Switzerland and Germany.

Participants 131 patients were screened for seasonal allergic rhinitis and 125 patients were randomised (butterbur 61; cetirizine 64).

Interventions Butterbur (carbon dioxide extract tablets, ZE 339) one tablet, four times daily, or cetirizine, one tablet in the evening, both given for two consecutive weeks.

Main outcome measures Scores on SF-36 questionnaire and clinical global impression scale. Results Improvement in SF-36 score was similar in the two treatment groups for all items tested hierarchically. Butterbur and cetirizine were also similarly effective with regard to global improvement scores on the clinical global impression scale (median score 3 in both groups). Both treatments were well tolerated. In the cetirizine group, two thirds $(8 / 12)$ of reported adverse events were associated with sedative effects (drowsiness and fatigue) despite the drug being considered a non-sedating antihistamine.

Conclusions The effects of butterbur are similar to those of cetirizine in patients with seasonal allergic rhinitis when evaluated blindly by patients and doctors. Butterbur should be considered for treating seasonal allergic rhinitis when the sedative effects of antihistamines need to be avoided.
\end{abstract}

\section{Introduction}

Allergic rhinitis, whether seasonal or perennial, is characterised by sneezing, rhinorrhoea, obstruction of the nasal passages, conjunctival and pharyngeal itching, and lacrimation. Although the term hay fever is commonly used for seasonal allergic rhinitis, it is inappropriate because the symptom complex is neither produced by hay nor associated with fever. ${ }^{1}$ Allergic rhinitis is caused by the deposition of allergens (often pollen) on the nasal mucous membranes, resulting in a type I hypersensitivity reaction. ${ }^{2}{ }^{3}$

Butterbur (Petasites hybridus; butter dock, bog rhubarb, exwort) is an Asteraceae herbaceous plant native to Europe, northern Africa, and south western Asia. ${ }^{4}$ The leaves and roots of butterbur contain a mixture of eremophilan type sesquiterpenes (petasines). Extracts of butterbur have been used in bronchial asthma, smooth muscle spasms, and headache, ${ }^{5}$ and studies have shown that petasines inhibit the biosynthesis of leukotrienes, which may be associated with antispasmodic activity and anti-inflammatory action in type I hypersensitivity. ${ }^{6-8}$

The usual treatment for seasonal allergic rhinitis is antihistamines. These reduce rhinorrhoea and sneezing but are less effective for nasal congestion and may cause sedation and drowsiness. The availability of corticosteroid nasal sprays without prescription has increased their use by patients with allergic rhinitis. Similarly, antihistamines can be obtained over the counter for treatment of hay fever, and all may interact with alcohol and decrease driving ability. ${ }^{9}$ We conducted a randomised controlled trial of butterbur extract tablets (ZE 339) and a commonly used non-sedating antihistamine (cetirizine) to compare the effectiveness of these two treatments.

\section{Participants and methods}

All participants were outpatients attending four general medicine and allergy clinics between June 1999 and June 2000. All physicians were experienced in using the instruments of assessment and had training in use of the scales before the study to enhance intercentre and intracentre consistency. In each clinic, all assessments were made by the same person.

Study medication consisted of butterbur (petasites carbon dioxide extract ZE 339 standardised to $8.0 \mathrm{mg}$ of total petasine per tablet; one tablet, four times daily) or cetirizine (one $10 \mathrm{mg}$ tablet daily), as recommended by the manufacturers. Blinding was assured by the use of "double dummies." Each day, participants took five tablets, four of which contained either placebo or butterbur, and one contained either cetirizine or placebo, depending on the treatment group. The study was performed according to current European Union and International Conference on Harmonisation guidelines on good clinical practice and the Declaration of Helsinki on Human Rights. The study was approved by the relevant ethics committees in Germany and Switzerland.

\author{
Allergy Clinic, \\ Hochwangstrasse 3 , \\ CH-7302 \\ Landquart, \\ Switzerland \\ Andreas Schapowal \\ consultant in ear \\ nose, and throat \\ medicine \\ andreas.schapowal@ \\ freesurf.ch \\ BMJ 2002;324:1-4
}




\section{Diagnostic criteria for enrolment}

Items A, B, C, and D must be fulfilled for each patient. A: History of seasonal allergic rhinitis symptoms for at least one year

B: Presence of all the following symptoms: sneezing, rhinorrhoea, itching (nose or eyes), and

nasal congestion

C: Severity of at least two of above symptoms must be rated $\geqslant 2$, where $0=$ none, $1=$ mild, $2=$ moderate,

$3=$ severe, and $4=$ very severe

D: Clinical presence of oedema of the nasal concha and pathologically increased nasal secretion

\section{Participants}

All participants were aged $\geqslant 18$ years, had a history of seasonal allergic rhinitis for at least two consecutive years, and fulfilled the seasonal allergic rhinitis diagnostic criteria (box). We excluded patients who had a history of alcohol or substance abuse; were pregnant or breast feeding; had parasitic disease causing increase in IgE or eosinophil levels; had taken corticosteroids in the past two months, antihistamines in the past six weeks, or anti-inflammatories in the past two weeks; had perennial (non-seasonal) rhinitis; had received an organ transplant; or had serious concomitant disease.

Baseline assessment was made at the referral consultation, when the inclusion and exclusion criteria were checked. All participants had skin allergy tests, and all but one were allergic to pollen, most commonly grass pollen. They also had a full medical examination and laboratory tests (haematology, biochemistry, pregnancy test), after which they were given enough treatment for two weeks. The acute nature of the illness meant an initial placebo phase was not appropriate. Participants could return after one week if they experienced adverse events or deterioration. At the visit at the end of week 2, participants had a full medical examination and laboratory tests (haematology, biochemistry) and we checked compliance and adverse events. Exposure to pollen was confirmed for each participant through crosschecking the treatment period with the online regional pollen count service (www.pollenallergie.de).

\section{Statistics, assignment, and analysis}

All data were processed and analysed by the University of Giessen's department of medical information technology. Randomisation was provided centrally in blocks of four. Analysis was on an intention to treat basis, defined as all randomised patients who had at least one baseline and one follow up value and took any medication. The planned sample size was a minimum of 120 patients, based on previous studies of allergic rhinitis, ${ }^{11}$ with a $10 \%$ expected withdrawal rate and an assumed effect size of 0.5 .

The main efficacy variable was change from baseline to end point in the score of each item on the medical outcome health survey questionnaire (SF-36), a validated tool for patient self assessment in this condition. ${ }^{11}$ Questions are grouped hierarchically in eight categories with a total score range of $0-100$ per item, and the questionnaire also includes one category with a five point score for comparing severity of the condition with that in the previous year.The secondary efficacy variable was the physicians' clinical global impression score. ${ }^{12}$ The hypothesis was that butterbur was not inferior to cetirizine at the end point, defined as within $10 \%$ of the SF-36 score or one point in the clinical global impression score.

The analysis followed a sequential confirmatory method of testing the hierarchically ordered items of SF-36 regarding changes in individual scores, whereby the main group is declared inferior when an item shows inferiority at any point during the testing sequence. The experimental probability of type 1 error is $\alpha=0.05$; one sided, with $80 \%$ power (with a minimum sample size of 50 patients per group). The null hypotheses were tested in a hierarchical sequence of rejecting hypotheses, whereby the procedure stops if the null hypothesis is accepted in the previous step. Values were calculated with $95 \%$ confidence intervals. Non-inferiority was tested by the Mann-Whitney rank sum test (one sided), and between treatment comparisons were tested by the Mann-Whitney test (two sided). We used the Shapiro-Wilk test for non-normally distributed data. The exploratory secondary variables were evaluated by inference statistics with a shifted null hypothesis adjustment to baseline according to the method of Abt, ${ }^{13}$ with means, standard deviation, medians, 95\% confidence intervals, minima-maxima, and absolute and relative frequencies. For participants who withdrew we carried forward the last observation. The mean of both treatment groups was used to substitute missing values.

\section{Results}

Patients' characteristics and flow through study A total of 131 patients were initially screened, and 125 were randomised (figure). Participants' characteristics at entry were similar in the two groups (table 1). The population was representative of patients with seasonal allergic rhinitis who seek treatment in the primary care sector, two thirds of patients being women and one third being smokers. All four main symptoms of the illness were moderately severe or worse in most participants (table 1). Rhinorrhoea was most commonly reported as moderately severe or worse, with sneezing, nasal congestion, and itchy eyes or nose having similar frequency and severity in both treatment groups. About half of patients thought their condition was worse than in the previous year.

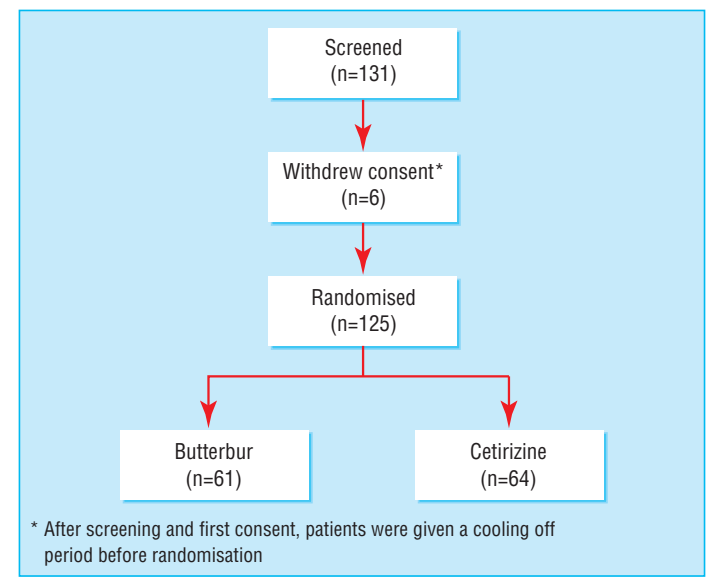

Enrolment of patients into study 


\section{Efficacy results}

Table 2 shows the results at the end of the treatment period. Analysis of the main outcome measures rejected the hypothesis of butterbur being inferior to cetirizine, with none of the scores in the butterbur group being more than $10 \%$ worse than in the cetirizine group. Analysis of the second outcome measures also showed no difference in efficacy between the two treatments. Exploratory analyses for between groups comparisons did not suggest a difference between the two treatments, although there were some trends in favour of the butterbur group.

\section{Safety results}

The overall incidence of adverse events was similar for the two treatments (table 3). No event could be considered to be typically associated with butterbur, all having been reported once or twice only. Conversely, two thirds of events in the cetirizine group were typical of antihistamines - that is, drowsiness and fatigue. One patient was withdrawn (butterbur group) because she required corticosteroids for previously existing asthma.

\section{Discussion}

Our randomised, double blind study showed that the effects of butterbur (ZE 339 extract tablets) are similar to those of cetirizine in patients with seasonal allergic rhinitis. Butterbur did not produce the sedative effects associated with antihistamines and was well tolerated by patients. The effects of this herbal treatment were confirmed by both patients and doctors.

\section{Methodological issues}

Although seasonal allergic rhinitis is common, methodologically robust studies are difficult to conduct, not least because of the easy access to anti-allergic treatments by patients. These treatments include a large number of antihistamines and corticosteroid nasal sprays available without prescription. To overcome the possible contamination of results by the use of other treatments, we monitored patients closely
Table 1 Patients' characteristics at entry to study

\begin{tabular}{lcc} 
Characteristic & Butterbur (n=61) & Cetirizine (n=64) \\
\hline Demographics: & $39(12)$ & $35(14)$ \\
\hline Mean (SD) age (years) & $1.72(0.08)$ & $1.71(0.09)$ \\
\hline Mean (SD) height (m) & $71(14)$ & $69(15)$ \\
\hline Mean (SD) weight (kg) & $38(62)$ & $46(72)$ \\
\hline No (\%) of women & $20(32)$ & $24(37)$ \\
\hline No (\%) of smokers & & \\
\hline Diagnostic characteristics: & $47(77)$ & $51(79)$ \\
\hline Sneezing (No (\%) moderate or worse) & $56(92)$ & $55(86)$ \\
\hline Rhinorrhoea (No (\%) moderate or worse) & $43(71)$ & $50(76)$ \\
\hline Itchy nose or eyes (No (\%) moderate or worse) & $45(74)$ & $82(21)$ \\
\hline Nasal congestion (No (\%) moderate or worse) & & $75(39)$ \\
\hline Mean (SD) SF-36 item score (main outcome measures): & $78(19)$ & $52(26)$ \\
\hline Physical function & $77(38)$ & $70(20)$ \\
\hline Emotional function & $54(23)$ & $60(22)$ \\
\hline Vitality & $72(20)$ & $46(40)$ \\
\hline Mental health & $60(24)$ & $70(26)$ \\
\hline General health & $46(39)$ & $71(25)$ \\
\hline Physical activity & $71(24)$ & \\
\hline Social functioning & $69(22)$ & \\
\hline Pain & & \\
\hline Secondary outcome measures: & $5.6(0.8)$ \\
\hline Mean (SD) clinical global impression score (severity of & \\
\hline condition) & $27(44)$ & \\
\hline SF-36 score for overall status (No (\%) worse or much worse & \\
than 1 year ago) & & \\
\hline
\end{tabular}

and allowed them to visit the clinics whenever they felt their condition needed further intervention. To enhance patient compliance, and bearing in mind the acutely debilitating symptoms of hay fever, we also kept the treatment period as short as possible (two weeks). In our experience, patients with this condition do not tolerate ineffective treatments for longer periods.

We considered that the comparator treatment should be a non-sedating antihistamine as these are the first choice of treatment for seasonal allergic rhinitis. We also excluded the use of corticosteroids of any kind from two months before and during the study. We then formulated the hypothesis of whether this herbal treatment was inferior to the antihistamine prospec-

Table 2 Results of primary and secondary outcome measures after two weeks' treatment

\begin{tabular}{|c|c|c|c|c|c|}
\hline & \multicolumn{2}{|c|}{ Median score (minimum-maximum) } & \multirow{2}{*}{$\begin{array}{l}\text { Median of } \\
\text { differences }\end{array}$} & \multirow{2}{*}{$\begin{array}{l}\text { P value for } \\
\text { comparison } \\
\text { between } \\
\text { medians* }\end{array}$} & \multirow{2}{*}{$\begin{array}{c}P \text { value for } \\
\text { non-inferiority test }\end{array}$} \\
\hline & Butterbur ( $n=61)$ & Cetirizine ( $n=64)$ & & & \\
\hline \multicolumn{6}{|l|}{ Primary outcome measures (SF-36 score) } \\
\hline Physical function & $95(50-100)$ & $95(15-100)$ & 0 & 0.75 & 0.001 \\
\hline Emotional function & $100(0-100)$ & $100(0-100)$ & 0 & 0.89 & 0.001 \\
\hline Vitality & $65(15-95)$ & $60(5-95)$ & 5 & 0.17 & 0.001 \\
\hline Mental health & $80(28-100)$ & $84(24-100)$ & 0 & 0.50 & 0.001 \\
\hline General health & $72(30-100)$ & $67(25-97)$ & 7 & 0.29 & 0.001 \\
\hline Physical activity & $100(0-100)$ & $75(0-100)$ & 0 & 0.16 & 0.001 \\
\hline Social functioning & $87.5(50-100)$ & $87.5(0-100)$ & 0 & 0.15 & 0.001 \\
\hline Pain & $84(41-90)$ & $84(22-90)$ & 0 & 0.44 & 0.001 \\
\hline \multicolumn{6}{|l|}{ Secondary outcome measures } \\
\hline \multicolumn{6}{|l|}{ Clinical global impression score: } \\
\hline Severity of condition $\ddagger$ & $-3(-2--6)$ & $-3(-2--7)$ & 0 & 0.82 & 0.0001 \\
\hline Global improvement§ & $3(2-7)$ & $3(2-6)$ & 0 & 0.82 & 0.001 \\
\hline Risk to benefit§ & $4(0.25-4)$ & $4(0.33-4)$ & 0 & 0.79 & 0.001 \\
\hline $\begin{array}{l}\text { SF-36 score for overall status (No (\%) worse or } \\
\text { much worse than } 1 \text { year ago)§ }\end{array}$ & $7(11)$ & $8(13)$ & - & 0.20 & 0.001 \\
\hline
\end{tabular}

*Mann-Whitney test (two sided). Significant values mean the treatment effects are different.

†Mann-Whitney rank sum test (one sided). Significant values mean butterbur is not inferior.

¥Negative values represent improvement in scores.

$\S$ Results for these items are medians at endpoint not adjusted for baseline.

Note: Data are non-normally distributed for all items (Shapiro-Wilk: $P \leqslant 0.01$ ) 
Table 3 Adverse events and withdrawals from treatment

\begin{tabular}{lcc} 
& $\begin{array}{c}\text { Butterbur } \\
(\mathbf{n}=\mathbf{6 1})\end{array}$ & $\begin{array}{c}\text { Cetirizine } \\
(\mathbf{n}=\mathbf{6 4})\end{array}$ \\
\hline No of adverse events reported & 10 & 12 \\
\hline No (\%) of patients reporting events & $10(16.4)$ & $11(17.2)$ \\
\hline Adverse events*: & & \\
\hline Fatigue & 2 & 6 \\
\hline Drowsiness & 0 & 2 \\
\hline Headache & 2 & 2 \\
\hline Itchy eyes & 1 & 0 \\
\hline Flatulence & 0 & 1 \\
\hline Diarrhoea & 1 & 0 \\
\hline Asthma & 1 & 0 \\
\hline Pruritus & 1 & 0 \\
\hline Raised liver enzyme activity & 1 & 0 \\
\hline Gastric upset & 1 & 1 \\
\hline Events causing temporary treatment interruption & 2 & 5 \\
\hline Events causing withdrawal† & 1 & 0 \\
\hline
\end{tabular}

*Adverse events were mostly mild to moderate.

tOne patient had exacerbation of bronchial asthma requiring concomitant

treatment not allowed in the trial and so was withdrawn.

tively in the protocol and tested the hypothesis hierarchically so that the analysis would stop if any of the tested items of the patients' self assessment showed inferiority for the herbal drug.

The number of randomised controlled trials with herbal medicines has increased substantially recently. ${ }^{14-16}$ Herbal treatments are being used more often by doctors and, in our experience, are often requested by patients.

\section{Value of butterbur}

Although the effects of butterbur have been linked to its constituents, ${ }^{6-8}$ we set out to test whether its clinical effects in seasonal allergic rhinitis were comparable to those of antihistamines as judged separately and blindly by patients and their doctors. The results showed that the effects of the two treatments are similar. The trends in favour of butterbur in some measures need to be confirmed in future prospective trials. With regard to safety, butterbur was well tolerated and did not have the sedative effects associated with antihistamines. Fatigue and drowsiness accounted for two thirds of adverse events reported in the antihistamine group.

We have no data on whether the efficacy and tolerability of continued treatment with butterbur would be similar to antihistamines. However, treatment for this condition is usually relatively short, being restricted to peaks in pollen count during the spring and early summer. We believe butterbur should be considered for treating seasonal allergic rhinitis, particularly in cases where the sedative effects of antihistamines need to be avoided.

Members of the study group were E Schrader (coordinator, Pohlheim), G Senti (Zurich), G Kunze (Rottweil), R Schellenberg (Hüttenberg), and J Dudeck and R H Boedeker (data audit and statistics, University of Giessen).

Contributors: AS is the principal investigator and guarantor of the paper. Professor Dr J Dudeck (University of Giessen) was responsible for statistics and data management.
What is already known on this topic

Seasonal allergic rhinitis (hay fever) is common in countries with temperate climates.

Most patients have their symptoms treated for short periods, particularly during peaks in atmospheric pollen count

\section{What this study adds}

After two weeks, the effects of butterbur and cetirizine were comparable in patients with hay fever

Butterbur produced fewer sedating effects than cetirizine

Butterbur should be considered when the sedating effects of antihistamines must be avoided

Funding: Zeller AG (Switzerland) supplied study medication and sponsored the study through the Clinical Research Organisation Praxis Klinische Arzneimittelforschung, Pohlheim, Germany. The interpretation of the results was the prerogative of the principal investigator and the study group.

Competing interests: None declared.

1 Austen KF. Diseases of immediate type hypersensitivity. In: Harrison's principles of internal medicine. 9th ed. Tokyo: McGraw-Hill.1981.

2 Berman BA. Allergic rhinitis: mechanisms and management. J Allergy Clin Immunol 1988;81;5;980-4.

3 Knapp HR. Reduced allergen-induced nasal congestion and leukotriene synthesis with an orally active 5-lipoxygenase inhibitor. $N$ Engl J Med 1990;323:1745-8.

4 Royal Horticultural Society online science and education. Petasites hybridus. www.rhs.org.uk (accessed 28 November 2001).

5 Meier B, Meier-Liebi M. Drogenmonographie Petasites. In: Hänsel R, Keller K, Rimpler H, Schneider G, eds. Hagers Handbuch der pharmazeutischen Praxis. 5th ed. Berlin: Springer Verlag, 1994:81-105.

6 Brune K, Bickel D, Peskar BA. Gastro-protective effects by extracts of Petasites hybridus: the role of inhibition of peptido-leukotriene synthesis. Planta Med 1993;59:494-6.

7 Bickel D, Roder T, Bestmann HJ, Brune K. Identification and characterization of inhibitors of peptido-leukotriene-synthesis from Petasites hybridus. Planta Med 1994:60:318-22.

8 Thomet OAR, Wiesmann UN, Schapowal A, Bizer C, Simon HU. Role of petasine in the potential anti-inflammatory activity of a plant extract of petasites hybridus. Biochem Pharmacol 2001;61:1041-7.

9 British Medical Association and Royal Pharmaceutical Society of Great Britain. Antihistamines, hyposensitisation, and allergic emergencies. British National Formulary. London: BMA, RPRSGB, 1998.

10 Bousquet J, Bullinger M, Fayol C, Marquis P, Valentin B, Burtin B. Assessment of quality of life in patients with perennial allergic rhinitis with a French version of the SF-36 Health Status Questionnaire. J Allergy Clin Immunol 1994:94:182-8.

11 Bousquet J, Duchateau J, Pignat JC, Fayol C, Marquis P, Mariz S, et al. Improvement of quality of life by treatment with cetirizine in patients with perennial allergic rhinitis as determined by a French version of the SF-36 questionnaire. J Allergy Clin Immunol 1996;98:309-16.

12 National Institute of Mental Health: 028 CGI Clinical global impressions In: Guy W, ed. ECDEU assessment for psychopharmacology. Rockville, MD: NIMH, 1976:217-29.

13 Abt K. Descriptive data analysis: a concept between confirmatory and exploratory data analysis. Meth Inform 1987;26:77-88

14 Wheatley D. LI160, an extract of St John's wort versus amitriptyline in mildly to moderately depressed outpatients-a controlled 6-week clinical trial. Pharmacopsychiatry 1997;30(suppl):77-80.

15 Woelk H. Comparison of St John's wort and imipramine for treating depression: randomised controlled trial. BMJ 2000;321;536-9.

16 Schellenberg R. Treatment for the premenstrual syndrome with agnus castus fruit extract: prospective, randomised, placebo controlled study. BMJ 2001;322:134-7.

(Accepted 13 September 2001) 